\title{
THE INFLUENCE OF METABOLIC THERAPY ON CLINICAL AND HEMODYNA- MIC PARAMETERS IN PATIENTS WITH STABLE ANGINA
}

\author{
Varahabhatla Vamsi ${ }^{1}$, Maganty Virajitha ${ }^{1}$, Lihasenko Ivetta ${ }^{2}$ \\ ${ }^{1}$ Zaporizhzhiya State Medical University, Zaporizhzhiya, UKRAINE \\ ${ }^{2}$ Department of Internal Medicine, Zaporizhzhiya State Medical University. Zaporizhzhiya, UKRAINE
}

\section{ABSTRACT}

Aims: The objective of our study is to investigate the antianginal activity of meldonium and its influence on the hemodynamics and clinical parameters in patients with stable angina who fall under III-IV functional classes according to the classification of the Canadian Heart Association.

Methods: The study was carried out including 105 patients with stable angina pectoris who were in III-IV functional classes at the age of 42-72 years. The first group $(n=52)$ was administered the standard treatment (beta-blockers, aspirin, atorvastatin). The second group of patients $(n=53)$ were given a combination of basic therapy along with meldonium $10 \%$ solution of $5 \mathrm{ml}$ intravenously for 10 days then $1000 \mathrm{mg}$ per day orally for 10-12 weeks. Suitable healthy people were selected as the control group $(n=36)$.

Results: Patients with stable angina pectoris were characterized with the reduction of workload, double work, time loading on bicycle ergometer, increased specific and total peripheral vascular resistance, increased central sympathetic activity on heart, decreased vagal and humoral activity on heart rate. The analysis of the data indicated a decrease in the frequency of anginal attacks after treatment in the first group by $45 \%$ and reduced daily nitroglycerin requirement by $48 \%$. Combination therapy resulted in a decrease of the parameters by $71.8 \%$ and $76 \%$, respectively.

Conclusion: After the treatment in both groups, we detected an improvement in physical exertion tolerance, reduction in total peripheral vascular resistance and sympathetic activity of the heart. Combination therapy with meldonium significantly reduced the central smypathetic activity and peripheral vascular resistance, also increased the workload, run time compared to the basic therapy.

Keywords: Hemodynamics, stable angina, carnitine

\section{INTRODUCTION}

Cardiovascular diseases are the number one cause of death globally. In 2016, an estimated 17.9 million people died from cardiovascular diseases, representing $35 \%$ of all global deaths (1). Most of the cardiovascular diseases can be prevented by changing behavioral risk factors such as tobacco use, unhealthy diet and obesity, physical inactivity and alcohol abuse. Nowadays metabolic therapy is an important direction of treatment for ischemic heart diseases (2).

Angina pectoris is one of the life-threatening cardiovascular conditions effecting the global population. Four national cross-sectional health examination stu- dies found recently that among the United States, in 40-74 years of age population, the prevalence rate of stable angina pectoris is higher among the females than in males (2).

With the data obtained from several clinical studies and drug trials, meldonium was identified as an anti-ischemic drug with several indications in neurological degeneration, pulmonary system dysfunction and cardiomyocyte protection (3). It provides the biochemical protection of cells from the effects of ischemia by effecting the carnitine metabolism. Despite imbalance in the need of the myocardial oxygen supply and its delivery, cardiomyocytes remain viable and function actively (3). 
The mechanism of meldonium is well understood; being a partial inhibitor of fatty acids oxidation and an analog structurally to the precursor of the gamma-butyrobetaine (GBB) (4). It inhibits carnitine metabolism and biosynthesis by competing with GBB hyrdroxylase enzyme, that catalyzes the last step in carnitine biosynthesis pathway, also inhibiting kidneys' reabsorbtion (5). Meldonium's other effect is the inhibition the long chain fatty acids transportation through the mitochondrial membranes. This progresses in the complete transport of cytosolic ATP and further delaying the beta-oxidation of fatty acids. This prevents the mitochondrial accumulation of the unoxidized fatty acids and acetyl carnitine and acetyl coenzyme-A. The increased cytosolic fatty oxidation is a specific signal for the activation of glucose oxidation (5).

Reduction in carnitine leads to situmilation of its precursor GBB synthesis, simultaneously activating the nitric oxide (NO) synthase, possibly is responsible for meldonium's antianginal and vasospastic effect (5).

A study conducted by Dzerve et al. (6) demonstrated the efficiency of meldonium in combination with the standard therapy at doses of $500 \mathrm{mg}$, twice daily for the duration of 10-12 weeks. There was an improvement in the exercise tolerance in patients with stable angina and the positive dynamics were dose-dependent (6).

The data from different publishers around the globe, gave evidence that meldonium's efficiency in myocardial ischemia by improving the systolic function of the myocardial cells, the inhibition of the occurrence of myocardial hypertrophy and dilatation, increased contractility of the smooth muscles of arterioles, increased tolerance to stress, reduction of angina symptoms (7).

The aim of this study is to investigate the antianginal activity of meldonium and its influence on the hemodynamics and clinical parameters in patients with stable angina that were III-IV functional classes.

\section{MATERIAL AND METHODS}

The study was carried out with 105 patients (66 males and 39 females) whose conditions are defined as stable angina III-IV functional classes (FC) according to the classification of the Canadian Heart Association (CHA) and was approved by the local ethics committee of Zaporozhye State Medical University (8). The mean age was $60.0 \pm 0.88$ years. The diagnosis of stable angina along with differential diagnosis was made with the following parameters: anamnesis data, risk factors, data of objective examination and the results of examinations using laboratory and instrumental methods. 27 patients had medical history of myocardial infarction. All the patients were assigned to the basic therapy, including $\beta$-blockers, calcium antagonists, antiplatelet agents and statins. Patients were distributed into two groups. The first group consisted of 52 people, who received conventional therapy, whereas 53 patients in the second group received the basic treatment along with a combination of meldonium $5 \mathrm{ml}$ of $10 \%$ solution intravenously for 10 days then converted to oral $1000 \mathrm{mg}$ per day for 10-12 weeks. The course of treatment was 10-12 weeks. Suitable healthy people were selected as the control group $(\mathrm{n}=36)$.

The patients, who had stable angina pectoris FC IIIIV classified by CHA with a disease duration more than 1 year, were included in the study (8). All patients gave their consent to participate in the study. On the other hand, the patients represented with hypertension, heart failure, sino-auricular and atrioventricular blocks, diabetes, liver and kidney disorders were excluded from the study.

Antianginal effect was estimated with the help of a self-observation diary, with the number of angina attacks and the number of nitroglycerin tablets taken per day.

Assessment of peripheral hemodynamics was performed using computer diagnostic complex "REOKOM" developed in laboratory and computer diagnostic systems of Kharkov National Aerospace University. Among all parameters, Systolic blood pressure-SBP (mm Hg), Total peripheral vascular resistance-TPVR (din x sm-5 x sec), Diastolic blood pressure-DBP (mm $\mathrm{Hg}$ ), Specific peripheral vascular resistance-SPVR (relative units) and Heart rate-HR (bpm) were selected.

The measurement of cardiac rhythm was carried out using computer cardiographic systems "Cardiolabs 2000" HAI MÉDICA (Ukraine) (9). CARDIOLAB is a computer-aided echocardiographic complex intended for conducting a wide range of cardiographic studies. The most informative parameters were: the average value of RR interval - RR average ( $\mathrm{sec}$ ), variational span of RR intervals - VS (sec), mode (relative units) - Mo, amplitude of Mode - AMo (\%), index of vegetative balance - IVB (relative units), vegetative indicator rhythm 
- VIR (relative units), Baevskiy tension index - TI (relative units).

Bicycle ergometer test (BET) was performed using SECA CARDIOTEST 100 (HÖRMANN, German) (10). Among all parameters, workload - WL (W), max - heart rate at the threshold of tolerance - HR (bpm), blood pressure at the threshold of tolerance - BPmax ( $\mathrm{mm} \mathrm{Hg}$ ), loading time $\mathrm{T}$ ( $\mathrm{min}$ ), double work - DW were selected. DW was calculated as an indicator that indirectly reflects the oxygen consumption of the myocardium by the formula: DW = HRmax $\mathrm{x}$ BPmax/100.

When comparing the study groups student t test was performed. Numbers, percentages, arithmetic mean \pm standard deviation were used as descriptive statistics. A p-value $<0.05$ was set for statistically significance.

\section{RESULTS}

Analysis of the data indicates a decrease in frequency of anginal attacks after the treatment in the first group by $45 \%$ and reduced daily nitroglycerin requirement by $48 \%$. Combination therapy resulted in more significantly decrease of the parameters by $71.8 \%$ and $76 \%$, respectively $(\mathrm{p}=0.0007794, \mathrm{p}=0.008145)$ (Figure $1)$.

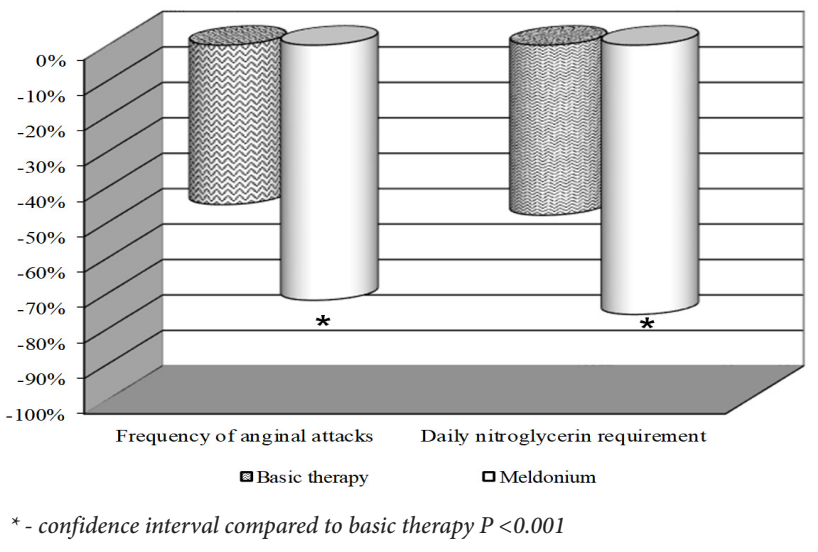

Figure 1: Dynamics of antianginal effect in patients after treatment.
In the second group, TPVR and SPVR decreased by $35 \%$ and $23 \%$ respectively than in the first group (Table $1)$.

Table 1: Dynamics of peripheral hemodynamics parameters in patients after treatment.

\begin{tabular}{|l|c|c|c|}
\hline \multicolumn{1}{|c|}{ Parameters } & Basic therapy & Meldonium & Healthy group \\
\hline & 1 & 2 & \\
\hline TPVR & $2159 \pm 93.8$ & $1859 \pm 36.2$ & $1846 \pm 114$ \\
& & $\mathrm{P}_{1.2}=0.0009668$ & \\
\hline SPVR & $51.7 \pm 2.57$ & $44.6 \pm 1.16$ & $44.5 \pm 2.74$ \\
& & $\mathrm{P}_{1.2}=0.000123$ & \\
\hline
\end{tabular}

${ }^{*} T P V R=$ Total peripheral vascular resistance, $S P V R=$ Specific peripheral vascular $r e$ sistance

Changes in heart rate are characterized by the following changes: VS variation rate increased by $11 \%$, AMo mode amplitude decreased by $7 \%$ in both groups, TI stress index decreased by $34 \%$ in the first group and $45 \%$ in the second group. This indicated a decrease in the central symphatetic activity, which led to a decrease in vascular tone, a decrease in peripheral resistance, and, as a consequence, an increase in exercise tolerance (Table 2).

Table 2: Dynamics of cardiac rhythm in patients after treatment.

\begin{tabular}{|l|l|l|l|}
\hline Parameters & Basic therapy & Meldonium & Healthy group \\
\hline & 1 & 2 & \\
\hline RR average (sec) & $0.815 \pm 0.032$ & $0.814 \pm 0.028$ & $0.804 \pm 0.013$ \\
\hline VS (sec) & $0.048 \pm 0.004$ & $0.047 \pm 0.006$ & $0.169 \pm 0.014$ \\
\hline Mo (relative units) & $0.831 \pm 0.028$ & $0.834 \pm 0.029$ & $0.816 \pm 0.027$ \\
\hline AMo (\%) & $53.5 \pm 2.02$ & $51.3 \pm 1.82$ & $49.5 \pm 1.68$ \\
\hline IVB (relative units) & $289.8 \pm 14.5$ & $\mathrm{P}_{1-2}=0.02780$ & \\
\hline VIR (relative units) & $7.53 \pm 0.500$ & $291.4 \pm 14.1$ & $285.3 \pm 20.1$ \\
& & $\mathrm{P}_{1-2}=0.00932$ & \\
\hline TI (relative units) & $215.5 \pm 17.4$ & $\mathrm{P}_{1-2}=0.833 \pm 1.13$ & $6.74 \pm 0.443$ \\
& & $201.7 \pm 12.1$ & $177.2 \pm 14.6$ \\
& & $\mathrm{P}_{1-2}=0.000244$ & \\
\hline
\end{tabular}

${ }^{*} R R$ average - the average value of $R R$ interval (sec), VS - variational span of $R R$ in tervals (sec), Mo - mode (relative units), AMo - amplitude of Mode (\%), IVB - index of vegetative balance (relative units), VIR - vegetative indicator rhythm (relative units), TI - Baevskiy tension index (relative units) 
Measurement on BET after treatment; the absolute magnitude of the power transferred to the threshold load was increased in patients of both groups. However, the addition of meldonium to conventional therapy contributed to a more substantial and a significant increase of WL compared with the isolated use of basic therapy ( $\mathrm{p}=0.00061)$. On the background of standard therapy, WL was increased by $42 \%$, SBP and HR at the threshold of tolerance by $6 \%$, while the growth of double work was $14 \%$, and the loading time was increased by $31 \%$ (Table 3 ).

Table 3: Comparison of bicycle ergometer results between healthy subjects and stable angina patients.

\begin{tabular}{|l|c|c|}
\hline Parameters & Healthy subjects & SA patients \\
& 1 & 2 \\
\hline WL (W) & $106.9 \pm 1.08$ & $45.8 \pm 1.16$ \\
& $137.6 \pm 1.28$ & $\mathrm{P}_{1-2}=0.00061$ \\
\hline HR max (BPM) & $183.8 \pm 1.87$ & $110.2 \pm 1.06$ \\
& & $\mathrm{P}_{1-2}=0.00301$ \\
\hline BP max (mmhg) & $262.1 \pm 2.09$ & $163.8 \pm 1.69$ \\
& & $\mathrm{P}_{1 \cdot 2}=0.02275$ \\
\hline Double work (DW) & $8.34 \pm 0.105$ & $169.8 \pm 2.27$ \\
& & $\mathrm{P}_{1-2}=0.00005$ \\
\hline T (min) & & $5.56 \pm 0.122$ \\
& & $\mathrm{P}_{1-2}=0.00543$ \\
\hline
\end{tabular}

The addition of meldonium increased WL by $59 \%$. The parameters HRmax and BPmax, as well as dual works were increased by $9.8 \%, 8 \%$ and $8.6 \%$, respectively and demonstrated a significant increase of $\mathrm{T}$ on bicycle ergometer at $49 \%$, which was close to that of the control group $(\mathrm{p}=0.00543)$. As the results in Figure 2 shows, the increase of $\mathrm{T}$ on bicycle ergometer was close to the control group. This leads to the functional enhancement of patients with coronary heart disease improving their tolerance to the physical stress and quality of life.

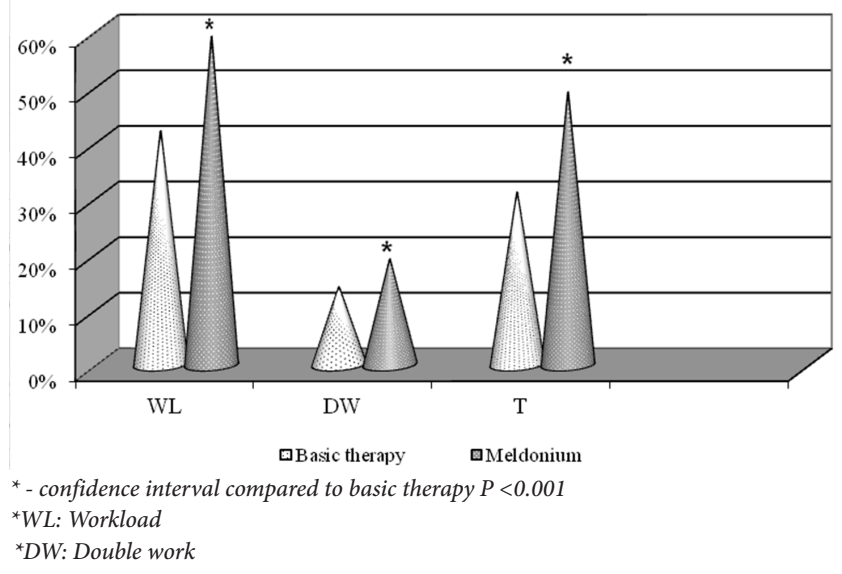

Figure 2: The change of bicycle ergometer test values in patients after treatment.

\section{DISCUSSION}

The modern literature highlights the favorable effects of meldonium on the cardiovascular system (11). Many authors pointed out the advantages of this drug indicating its antianginal, anti-arrhythmic effects, also its role in acid-base regulation in blood and NO metabolism (12). Sakharchuk et al. (13) pointed out that meldonium was an effective drug for the treatment of cardiac insufficiency due to ischemic heart disease. Their study on meldonium showed a positive effect on the blood hemodynamics, normalization of NO content and increased quantity of the membrane-bound erythrocytic enzymes.

Dudko et al. (14) indicated in their study that meldonium's effects against a placebo background in 50 patients revealed its antianginal effects and increased physical workload capacity in patients.

Ol'binskaia et al. (15) reported antianginal and anti-arrhythmic action of meldonium, by evaluating its ability to prevent ventricular extrasystoles, enhancement of physical capacity in the administered patient group.

Karpov et al. (16) revealed that the drug had a positive effect in improving the coronary circulation in patients with atherosclerosis.

Teplyakov et al. (17) demonstrated the anti-ischemic effects of meldonium in postinfarction left ventricular dysfunction patients in whom chronic hypoxia developed due to chronic pulmonary congestion, having an improved dynamic in their acid-base balance of their blood.

Enina et al. (18) reported that there was a transient decrease in the arterial blood pressure after 30 minutes of the drug administration in patients. The clinical parameters of blood changed after 60-90 minutes after bolus injection normalizing the cerebrovascular activity.

The obtained data from our study show that meldonium is capable of reserving additional ways of energy metabolism, contributes to an optimization of energy usage to facilitate the hemodynamic condition of heart. More studies on higher risk populations should be carried out to investigate the safety and efficacy of this drug proving its positive dynamics in patients with cardiovascular diseases. 
In conclusion the inhibitor of carnitine-dependent fatty acid oxidation, meldonium, strengthens the antianginal effect of basic therapy. It leads to the expansion of the functional capabilities of patients with IHD and increases their tolerance to the physical exertion. Meldonium contributes to a more pronounced decrease in central symphatetic activity system and the total peripheral resistance of the vessels. The course of treatment with meldonium improves antianginal effect of basic therapy and significantly reduces the total peripheral resistance in the vessels ( $p=0.0009668)$. Combination treatment of meldonium contributes to a more pronounced exercise test tolerance when compared with basic therapy.

Ethics Committee Approval: This study was approved by the local ethics committee of Zaporozhye State Medical University.

Informed Consent: Written informed consent was obtained from the participants of this study.

Conflict of Interest: The authors declared no conflict of interest.

Author contributions: Concept: GNV. Design: GNV. Supervision: GNV, VT. Resources: GNV. Materials: GNV. Data collection and/or processing: GNV, VT, VV. Analysis and/or Interpretation: GNV, VT, VV. Literature Search: GNV, VT, VV. Writing Manuscript: VT, VV. Critical Review: GNV, VT, VV.

Financial disclosure: The authors declared that this study received no financial support.

\section{REFERENCES}

1. World Health Organization. Cardiovascular diseases (CVDs). Available from URL:http://www.who.int/ news-room/fact-sheets/detail/cardiovascular-diseases-(cvds) (17/05/2017).

2. Centre for disease control. National Center for Health Statistics. National Health Interview Survey, 2016. (cited 2018 Sep 21). Available from URL: https://www. cdc.gov/nchs/nhis/shs/tables.htm.

3. Dzerve V, Matisone D, Pozdnyakov Y et al. Mildronate improves the exercise tolerance in patients with stable angina: results of a long term clinical trial. Seminars in Cardiovascular Medicine 2010;16:3.

4. Liepinsh E, Makarova E, Sevostjanovs E et al. Carnitine and $\gamma$-butyrobetaine stimulate elimination of meldonium due to competition for octn2-mediated transport. Basic Clin Pharmacol Toxicol 2017;120(5):450-6.
5. Lindquist C, Bjørndal B, Rossmann CR et al. A fatty acid analogue targeting mitochondria exerts a plasma triacylglycerol lowering effect in rats with impaired carnitine biosynthesis. PLoS ONE 2018;13(3):1-16.

6. Dzerve V, MILSS I Study Group. A dose-dependent improvement in exercise tolerance in patients with stable angina treated with mildronate: a clinical trial “MILSS I". Medicina (Kaunas) 2011;47(10):544-51.

7. Zhernakova NI, Olesya V, Romaschenko et al. The importance of the smoking factor in personalized complex pharmaco- therapy of ischemic heart disease with the use of metabolic correctors. International Journal of Advanced Biotechnology and Research 2018;9(1):1016-21.

8. Canadian Cardiovascular Society. "Canadian Cardiovascular Society grading of angina pectoris". (cited 2018 Sep 25). Available from URL: http://www.ccs.ca/ images/Guidelines/Guidelines_POS_Library/Ang_ Gui_1976.pdf.

9. Electrocardiographic complex CARDIOLABS. (cited 2018 Sep 25). Available from URL: http://xai-medica.com/cardiolab+/.

10. Veloergometer SECA CARDIOTEST. (cited 2018 Sep 24). Available from URL: http://www.esus.ru/php/ content.php?id=3134.

11. Danilenko LM, Klochkova GN, Kizilova IV et al. Metabolic cardioprotection: new concepts in implementation of cardioprotective effects of meldonium. Research result 2016;2(3):95-100.

12. Zhu Y, Zhang G, Zhao J et al. Efficacy and safety of mildronate for acute ischemic stroke: a randomized, double-blind, active-controlled phase II multicentert trial. Clin Drug Investig 2013;33:755.

13. Sakharchuk II, Denisenko GT, Grushko VS et al. The use of mildronat in heart failure in patients with chronic ischemic heart disease. Vrach Delo 1989;9:213.

14. Dudko VA, Koshel'skaia OA, Sokolov AA. The use of mildronat with stenocardia patients. Vrach Delo 1989;10:64-7.

15. Ol'binskaia LI, Golokolenova GM. Use of mildronate in cardiac insufficiency in patients with ischemic heart disease. Klin Med (Mosk) 1990;68:39-42. 
16. Karpov RS, Dudko VA, Shipulin VM et al. The clinical instrumental evaluation of treatment efficacy in patients with concomitant atherosclerosis of the coronary, cerebral and peripheral arteries. Ter Arkh 1991;63:903.

17. Teplyakov AT, Sankevitch TV, Stepatcheva TA et al. The use of fatty acid beta- oxidation inhibitor mildronate as monotherapy or incombination with atenolol in patients with left ventricular dysfunction after myocardial infarction. Kardiologiia 2003;43:15-8.

18. Enina G, Timofeeva T, Egere D et al. Medicinal effects and indications to mildronate application in neuroangiologic practice. Experimental and Clinical Pharmacotherapy 1991;19:164-71. 